\title{
Droplet Formation and Settlement of Phase-Change Ink in High Resolution Electrohydrodynamic (EHD) 3D Printing
}

\author{
Yiwei Han, Chuang Wei and Jingyan Dong \\ Department of Industrial and Systems Engineering, North Carolina State University, Raleigh, NC \\ jdong@ncsu.edu
}

\begin{abstract}
This paper presents a modeling framework to model the droplet formation and settlement on substrate of phase-change ink in high resolution electrohydrodynamic (EHD) printing process, which can successfully produce sub $10-\mu \mathrm{m}$ droplet footprints and $3 \mathrm{D}$ microstructure. We have used Finite Element Analysis (FEA) to develop the model for droplet formation and droplet settlement. Two important competitive forces in EHD printing, electrostatic force and surface tension force, are modeled by FEA. The droplet size is obtained by balancing the electrostatic force and surface tension of the pending droplets at the tip of the meniscus under different printing conditions. With the results from FEA analysis about the charge on a droplet and electrostatic field distribution, the droplets inflight velocity and impact velocity on the substrate are derived numerically. With the derived impact velocity, the droplet spreading and settlement on the substrate is also modeled by FEA. The results from FEA models are compared with the experimental measured droplet dimensions at different process conditions to validate the developed model, which demonstrate very good agreement between the experimental results and model prediction. We have successfully applied EHD printing process for phase-change wax material, which is widely used in 3D printing or additive manufacturing for supporting and model material, to achieve high resolution sub $10-\mu \mathrm{m} 3 \mathrm{D}$ structures.
\end{abstract}

Keywords: Electrohydrodynamic printing, FEA modeling, 3D printing, Phase-Change Ink

\section{Introduction}

Additive manufacturing (Beaman 1997, Gibson 2009, Hutmacher 2004, Kruth 1998, Melchels 2011) is capable of rapid prototyping and small volume part production, which have been applied in many applications (e.g. aerospace, automotive, medic device, electric device). Traditional additive manufacturing including stereolithography, selective laser sintering, electron beam melting, 3D printing, and fused deposition modeling (Gibson 2009, Kruth 1998), have their best resolution no 
better than $50 \mu \mathrm{m}$. The limitations of traditional additive manufacturing processes are intrinsic and very difficult to be improved. For example, in laser or electron beam melting based processes, the fabrication resolution is limited by many factors, including beam spot size, thermal diffusion, and power size. For printing or extrusion based processes, the resolution is primarily limited by the size of the printing nozzle. Scaling down the nozzle size will require impractically high extrusion pressure for most liquid phase inks, since extrusion pressure scales up much faster when the nozzle diameter is decreased.

High-resolution additive manufacturing is important for many engineering applications. Bad part accuracy and surface finish significantly limit the application of current additive manufacturing processes in the production of high precision industrial parts. A high resolution 3D printing process with micron-scale accuracy and high quality surface finish can effectively reduce the time and cost in post-processing. High precision industrial parts need high precision micro-scale part with high surface finishing as to reduce the complexity of post-processing. In tissue engineering scaffolds, structure with micro-scale size similar with cells has many advanced functions to regulate cell responses to the scaffold (Tan 2002, Wang 2012). Given the resolution limitation of the existing additive processes, new printing methods need to be developed to meet the high-resolution requirements for additive manufacturing,

Electrohydrodynamic (EHD) printing (Mishra 2010, Park 2007, Poellmann 2011) is a high resolution printing approach, in which the ink is subjected to electrostatic field to form a Taylor-cone and produce a fine jet or droplet from the cone. Since the produced jet/droplet diameter is significantly smaller than the nozzle size, EHD printing can overcome the resolution limitation from the nozzle size in traditional 3D printing. Currently, most of the EHD printing applications focus on the 2D pattern with only some initial investigation in 3D structure fabrication (Wei 2013, Wei 2014). Drop-ondemand EHD printing with using phase change ink would provide a promising process for micro-scale 3D structure fabrication. To achieve reliable process control and planning, it is very important to understand the mechanism of the EHD printing process and developing a reliable process model. However, it is still very challenging to predict the droplet formation (Collins 2008, Collins 2013) and droplet size for EHD printing at different process conditions. Computational modeling can provide promising result on droplet formation in electrospray (Collins 2008, Collins 2013, López-Herrera 2011, Forbes 2010), but due to the complex coupled physics (electrostatic force, fluid dynamics, and singularity from droplet pinch-off) of EHD printing, so far, there is no commercially available numerical solver that can be successfully applied to EHD drop-on-demand printing. Some groups (Collins 2008, Collins 2013, López-Herrera 2011, Forbes 2010) have developed their own solver to study some sub-set problem in EHD printing process with lots of computational load. Few of them have constructed a highly simplified model just to provide the insight of this process from engineering perspective (Han 2014, Kim 2010, Lee 2013, Wei 2014).

In this work, we have applied EHD printing for phase-change ink (wax) for micro-scale 3D printing of sub-10 micron structures. The droplet formation, size, droplet impact and settlement on the substrate in EHD printing is modeled by Finite Element Analysis (FEA) with respect to different process conditions. The model for the overall printing process is divided into two sub-models. First, FEA is used to develop the model for droplet formation and in-flight speed before impact substrate. Two important forces in EHD printing, electrostatic force and surface tension force, are modeled by FEA. The droplet size is obtained by balancing these two competing forces on the pending droplets at the meniscus tip for different printing conditions. After calculating the overall charge on a droplet and electrostatic field distribution between the nozzle and substrate from FEA analysis, the droplets inflight velocity and impact velocity on the substrate are derived numerically. Then, using the derived impact velocity, the droplet spreading and settlement on the substrate is modeled by ANSYS Fluent. The results from FEA models are compared with the experimental measured droplet dimensions to validate the developed model, which demonstrate very good agreement between the experimental results and model prediction. We have successfully applied EHD printing process for phase-change 
wax material, which is widely used in 3D printing or additive manufacturing for supporting and model material, to achieve high resolution sub $10-\mu \mathrm{m} 3 \mathrm{D}$ structures.

\section{Electrohydrodynamic Printing System and Process}

The material used in this EHD printing process is wax (as a phase-change ink) with a melting point of $\sim 55^{\circ} \mathrm{C}$, which will be solidified quickly once it is printed onto the substrate. Our printing system consists four parts: a three-axis (XYZ) precision stage, a thermal control system, a high voltage supply, and a pneumatic dispensing system, which are shown in figure 1 . The motion stage is installed on a vibration isolated table. Three linear stages are configured in $\mathrm{XYZ}$ directions and are used to move the printing head to the programmed locations. A high-resolution camera is used to monitor and record the printing process. The pneumatic dispensing system provides backpressure to the nozzle for control the liquid material flow rate. The syringe temperature is set to $110^{\circ} \mathrm{C}$ for melting wax and reduces wax surface tension and viscosity. The nozzle with 50 $\mu \mathrm{m}$ orifice and 150 outer-diameter is used in the EHD printing processes. The gap between nozzle and the substrate is chose about $130 \mu \mathrm{m}$, which gives the best performance for the printing processes. A function generator (Agilent 33220A) was used to generate the voltage command, which was then amplified by a voltage amplifier (Trek 610A) to be used for EHD-jet printing. The printed patterns and 3D structures are characterized by using optical microscope and SEM.

In EHD-jet printing, a large voltage is applied between the nozzle tip and substrate, which causes mobile ions in the printed ink (melted wax in this study) to gather near the surface at the nozzle tip. The electrostatic force deforms the meniscus at the nozzle end into a conical shape (i.e., Taylor cone). With large enough electric field, the electrostatic force from surface charge repulsion at the cone apex exceeds the surface tension of the meniscus, and a droplet or a jet of ink is printed from the Taylor cone onto the grounded substrate. The fluid properties of the printing material, along with the process conditions, mostly the applied voltage determines the plotting results.

In our experiment, for melted wax in EHD printing, as we gradually increased the voltage, a meniscus and Taylor cone was gradually forming at the tip of the nozzle (Figure 2(a)). When the applied voltage is large enough (around $700 \mathrm{v}$ to $750 \mathrm{v}$ ), micro droplets were ejected from the Taylor cone and printed onto the substrate. When the voltage is too large, satellite droplets are printed, indicating unstable printing condition. From Figure 2, clearly increasing the voltage will decrease droplet size from $17 \mu \mathrm{m}$ to $8 \mu \mathrm{m}$ and increase ejection/printing frequency from $5 \mathrm{~Hz}$ to $19 \mathrm{~Hz}$, which can be easily explained by the mechanism of EHD printing. A
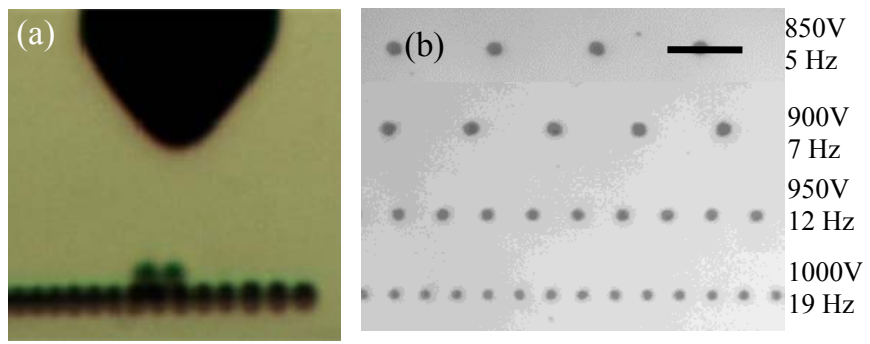

Figure 2: (a) Pulsating mode of EHD printing of wax. (b) Droplet size and printing frequency at positive voltages (Droplet sizes from top to bottom: $17 \mu \mathrm{m}, 14 \mu \mathrm{m}, 11 \mu \mathrm{m}, 8 \mu \mathrm{m}$ ) 
higher voltage or electrical field strength will increase surface charge density at the Taylor cone. As a result, droplets with smaller volume can obtain large enough electrostatic force to overcome surface tension to be ejected from the cone tip. The high electric field also increases the charge migration rate. As a result, it takes less time to accumulate enough charge at the apex to initiate ejection, which increases the printing frequency.

\section{EHD Printing of 3D Microstructures}

We have successfully fabricated high aspect of ratio micro-scale 3D structures using EHD printing process with phase-changed ink. The 3D micro-structures were drop-on-demand printed layer by layer, following the programmed sequence. Due to the micro-scale size of the droplets, the ejected melted wax droplets solidify quickly after printed onto the substrate, thus enables layered manufacturing of 3D structures, as shown in Figure 3. Complex continuous 3D structures can be printed by coordinating the ejection speed and plotting speed to achieve the required overlap between the neighboring droplets. With proper overlap between droplets, continuous features can be fabricated with very smooth edge. Repeating the printing sequences layer by layer, 3D structures can be printed directly with good surface finish. Figure 3 (a) and (b) demonstrate the 3D structures of a circular tube. The height of those structures is about $40 \mu \mathrm{m}$ to $60 \mu \mathrm{m}$, with a wall thickness of $\sim 6 \mu \mathrm{m}$. This printing resolution is one or two magnitude better than the capability of traditional 3D printing. High aspects of ratio pillars are directly printed with a diameter similar to the each single droplet diameter. Droplet will printed on top of the previous droplet once the nozzle is fixed at a certain location. Figure 3(c) (d) shows an EHD printed pillar. The diameter of pillars are $\sim 15 \mu \mathrm{m}$, and the maximum height of those pillars can reach to $\sim 100 \mu \mathrm{m}$, which will give an aspect of ratio of $\sim 7$.

Currently the structure height of our EHD 3D printing configuration is limited by the distance between nozzle and substrate. In our printing system, the ground electrode is located under the substrate. When the printed structures are close to the nozzle, the electric field is distorted, which cause unstable printing with uncontrollable printing speed and droplet diameter. However, this height

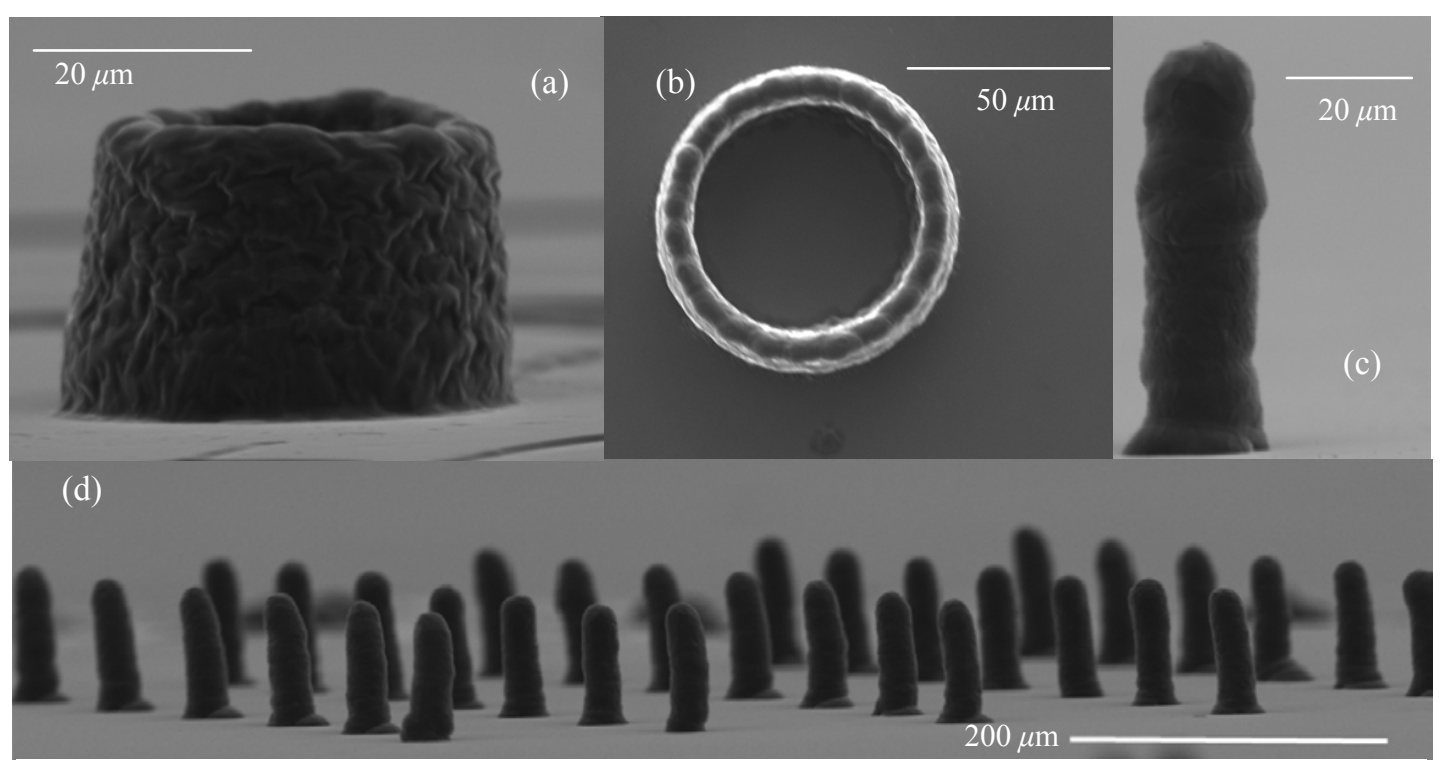

Figure 3: Micro-structures printed from EHD printing process.(a,b) circular tube with thin walls. (c) Close view of a single pillar. (d) Micro-pillar array matrix. 
limitation can be solved by using new printing head design that integrate ground electrode into the printing head.

\section{Modeling of Electrohydrodynamic Printing Process}

Modeling the EHD-jet printing process is essential for process control. It is valuable to predict the droplet dimension at different system configurations and process conditions for planning the printing sequences. Due to highly complex and coupled physics of the EHD printing process, it is extremely difficult to obtain the analytical solution to describe the EHD printing behaviors. In this paper, instead of studying the complex coupled electrical and fluidic behavior together, we separated the printing process into two stages and developed their models individually. The first stage is droplet formation and in-flight to substrate under electrostatic force, in which two important forces (electrostatic force, and surface tension force) in EHD printing are modeled by FEA using ANSYS APDL. The second stage is the droplet impact and settlement on the substrate, which is modeled by ANSYS Fluent.
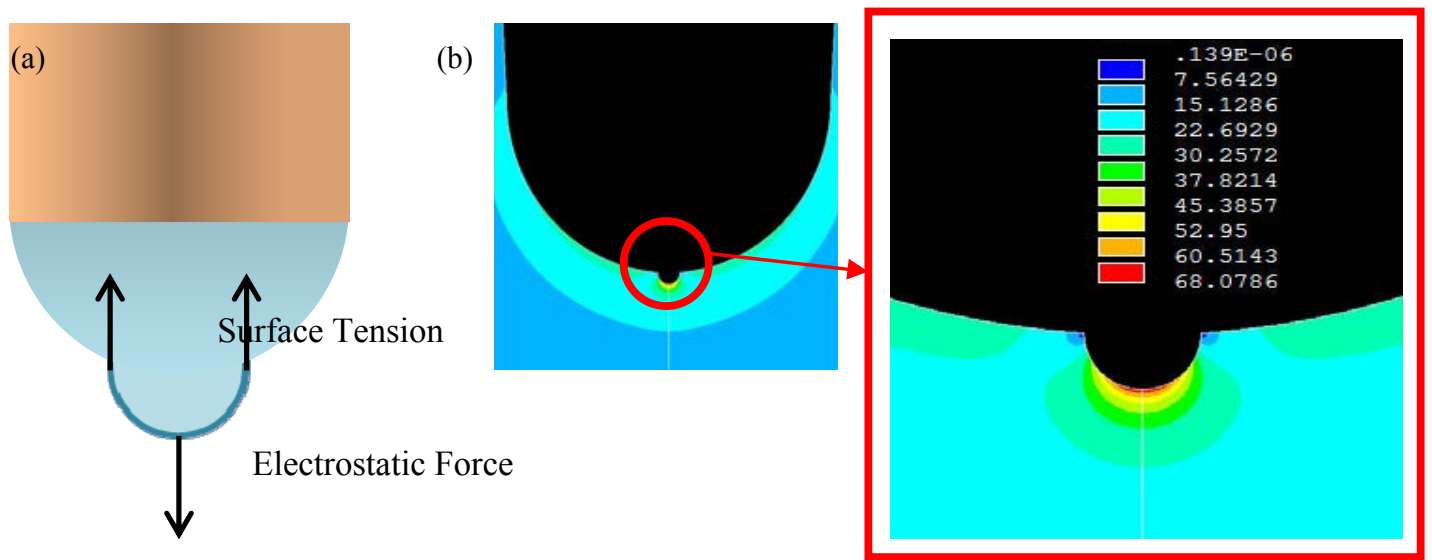

Figure 4: Schematic configuration for FEA modeling of the electrostatic force on the droplets. b) Electrical field distribution around the nozzle tip during droplet ejection.

\subsection{Modeling for Droplet Formation and In-Flight Velocity}

To model the droplet formation, we assumed a hemispherical meniscus forms at the end of the nozzle tip, and the diameter of the hemispherical meniscus is same to the diameter of the nozzle tip. The scheme for FEA model is shown in Figure 4 (a). The EHD printing process depends on two competing forces, the electrostatic Columbic force, and surface tension force. When the electrostatic Columbic force exceeds the surface tension force, a droplet is ejected from the meniscus. In FEA analysis, we study a state that the pending droplet at the meniscus apex has a hemisphere shape. In this state, the droplet with the fixed diameter has the largest surface tension force, and will be ejected from the meniscus if the electrostatic force acting on the droplet surface is larger than the surface tension force.

Using the configuration in Figure 4, with FEA analysis, we can calculate the electrostatic force acting on the half-ejected droplet surface at different process conditions, such as voltage, droplet diameter, and the nozzle/meniscus diameter, as shown in Figure 5. The electrostatic force $F_{e}$, can be expressed by an empirical equation using power law fitting as $F_{e}=c V^{k 1} D_{d}{ }^{k 2} D_{N}{ }^{k 3}$ in which $F_{e}$ is a 

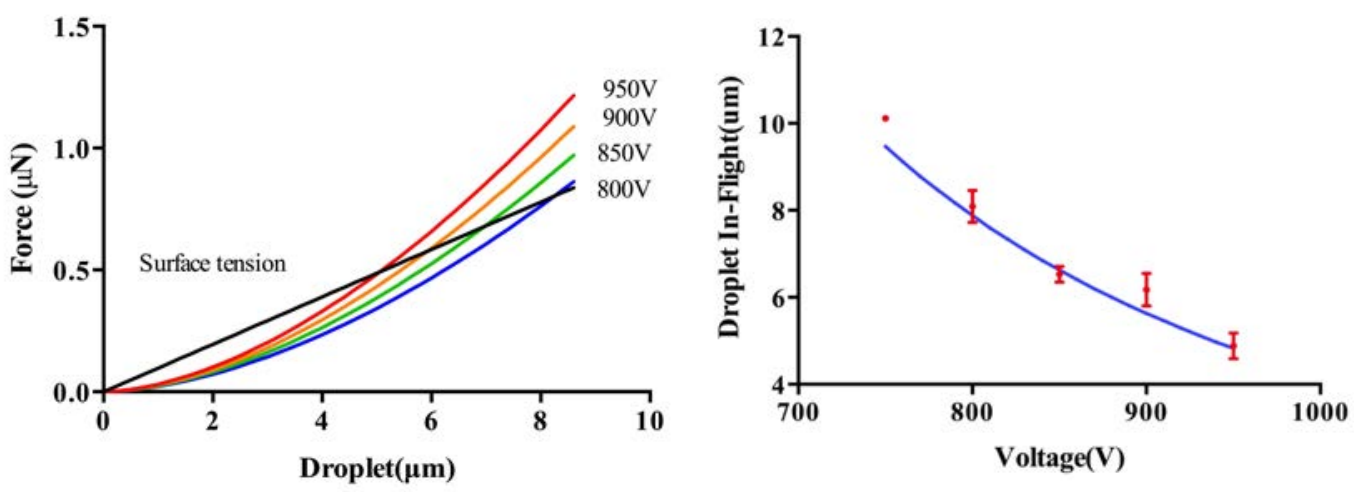

Figure 5: a) Intersection of electrostatic force and surface tension force on the droplet give the resulting droplets diameter at different voltages. b) Result of droplets in-flight dimension from FEA (blue line) and experimentally measured (red data points)

function of voltage $V$, droplet diameter $D_{d}$, and the nozzle/meniscus diameter $D_{N}$. By changing one parameter and keep other parameter constant at a time, we can calculate the value for $c, k 1, k 2$, and $k 3$ from FEA analysis, which provide the relationship between the electrostatic force and the process parameters. For the tip used in this study, the final equation is $F_{e}=3.46 e-6 V^{2.0} D_{d}^{1.702} D_{N}{ }^{-1.078}$.

In the EHD printing when the electrostatic force is larger than surface tension force, the sum of force will make droplet detach from the meniscus. The equation for surface tension force is $F_{s}=\sigma \pi$ $D_{d}$ Where $\sigma$ is surface tension coefficient of melting wax $(\mathrm{mN} / \mathrm{m})$. By connecting two equation together, we can get that $F_{s}=\sigma \pi D_{d}=F_{e}=c V^{k 1} D_{d}{ }^{k 2} D_{N}{ }^{k 3}$, and resulting droplet diameter $D_{d}$ as $D_{d}=\left(\frac{c V^{k 1} D_{N}{ }^{k 3}}{\pi \sigma}\right)^{\frac{1}{1-k 2}}$. The solution for the resulting droplets diameter can be solved graphically as in

Figure 5(a). When the line of the surface tension force intersects with the curve of the electrostatic force for a certain nozzle tip with applied voltages, the electrical static force is large enough to overcome the surface tension to produce a droplet with certain diameter. Using this method, for each voltage applied to the nozzle, we can estimate the corresponding droplet diameter in EHD printing at a specific process conditions.

Due to the phase-change ink (wax) used in EHD printing, we can directly measure the volume of printed wax droplets on the substrate, and validate the FEA model with experimental results. The droplets diameter predicted by the FEA model and the experimental results are plotted in Figure 5(b). The FEA results are in good agreement with the measured droplet dimensions with the maximum prediction error less than $10 \%$. The difference between FEA model and the experimental results may come from factors that include the measurement error of the nozzle diameter, the difference in the surface tension for different tips due to the temperature variation.

Droplet in-flight velocity, especially the droplet impact velocity is critical to study droplet impact/settlement on the substrate. In this study, an analytical model is developed to obtain the impact velocity of the droplets using the results from FEA analysis. The schematic of the droplet in-flight model are shown in Figure 6 (a). Two different forces are applied on the in-flight droplet, electrostatic force and viscous drag force. Electrostatic force accelerates the droplet velocity, and viscous drag force decelerates the velocity. The expression of electrostatic force is $F_{e s}=E q$, and the expression for drag force is $F_{d}=1 / 2 \rho v^{2} A C_{d}$, where $\rho$ is air density, $C_{d}$ is drag coefficient, and $v$ is the droplet velocity and $A$ is droplet cross-section area. The electrostatic field strength along the axial direction of the nozzle can be obtained from FEA analysis. An example of electrostatic field strength along the center axis is shown in Figure 6(b). The electrostatic field strength relies on the voltage difference between the nozzle and the substrate, which is largest around the nozzle tip and gradually decreases when the 

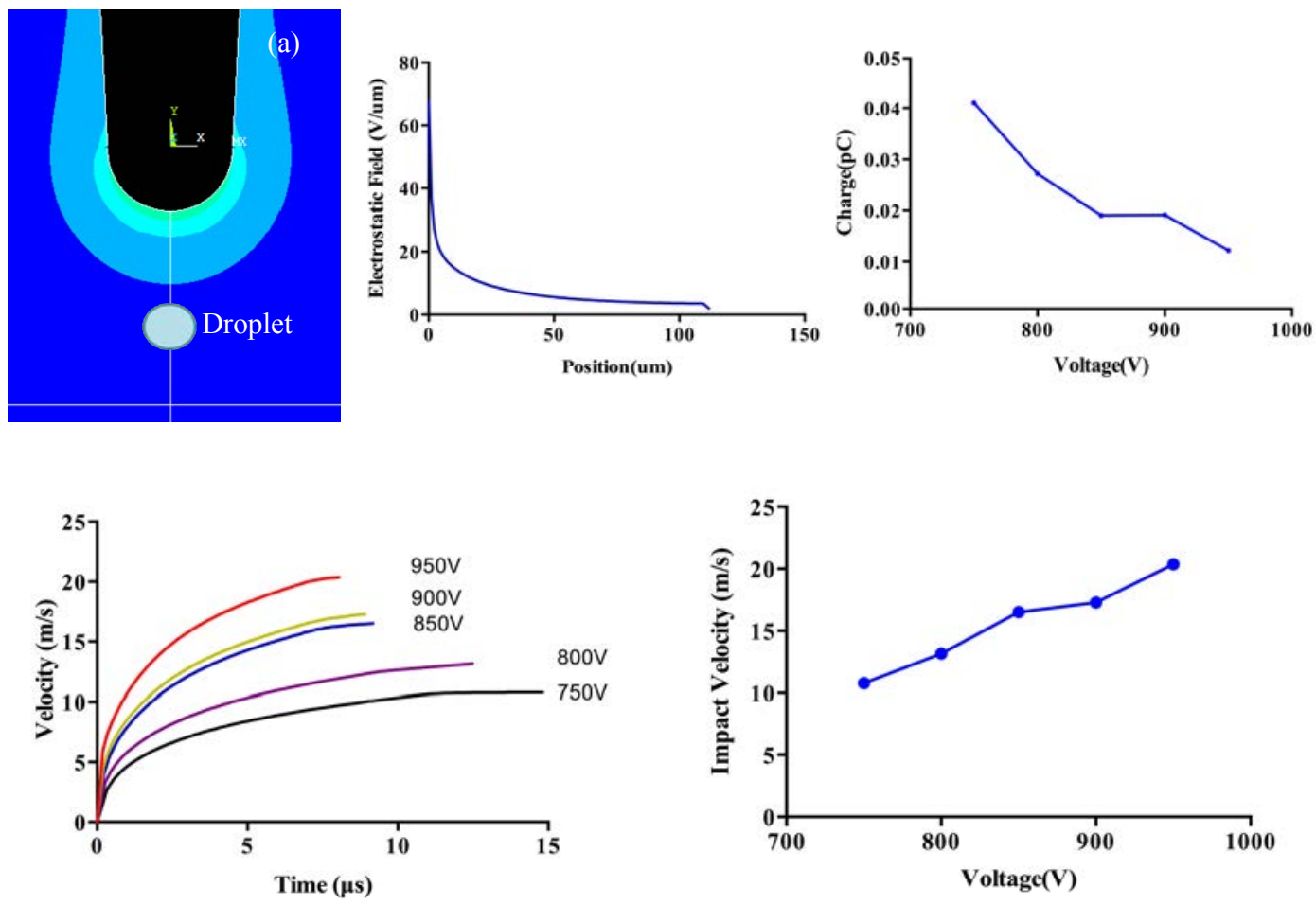

Figure 6: a) FEA model of droplet in-flight between tip and substrate. b) Electrostatic field distribution along center axis. c) The amount of charge of droplet under different voltages. d) Droplet velocity under different voltage and different flight time e) Droplet impact velocity with different printing voltages.

droplet moves to the substrate. The charge of the droplet is obtained from the charge accumulating on the ejecting hemisphere droplet, and is uniformly distributed on the droplets surface. Figure 6(c) shows the charge of each droplet at different process conditions. Then we can calculate the droplet inflight velocity using the electrostatic force and drag force. In our calculation, the gravity force of the droplet is extremely smaller (six-order of magnitude smaller) than other two force, thus the gravity effect can be neglected. After integrating the acceleration along z-axis, the velocity of the droplets can be derived as the function of time and travel distance. Figure 6 (d) shows that the velocity of droplet in-flight under different voltages and different flight time. When droplet finally impact on the substrate, it will get the largest velocity, which is the impact velocity and this velocities are shown in Figure 6(e).

\subsection{Modeling for Droplet Settlement on Substrate}

It is important but very diffuctlty to study the droplet impact and settlement on substrate surface theretically. Understanding this process will help us to predict the final droplet footprint on the substrate and the resulting shape for 3D fabrication. The ejected droplets only travel several microseconds before reaching the substrate. In the model, we use Anasys Fluent to study the droplet interaction with the substrate mechanically (by impact) and thermally (solidification). Droplet impact and solidfication can be divided into four typical stages: droplet in-flight befire impact, initial droplet impact, droplet spreading, and droplet solidfication, as shown in Figure 7. The initial stage in the model is that the droplet has reached the maximum inflight speed and ready to impact the substrate, 

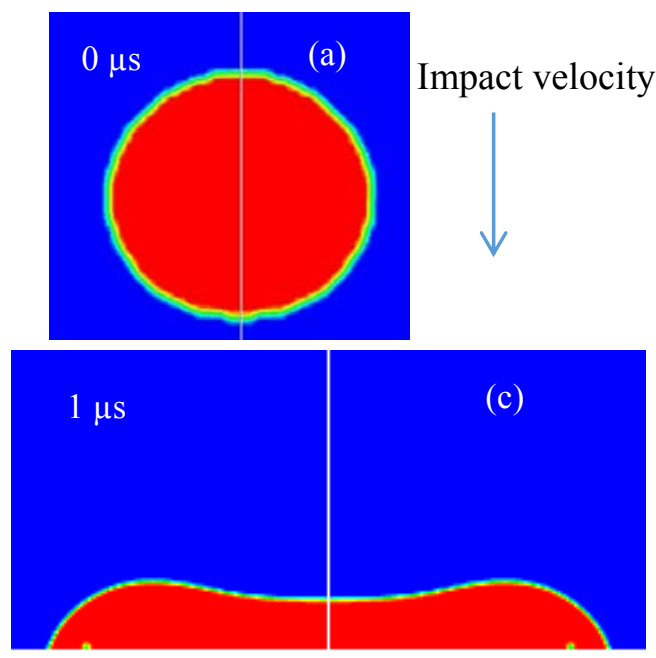

(e)

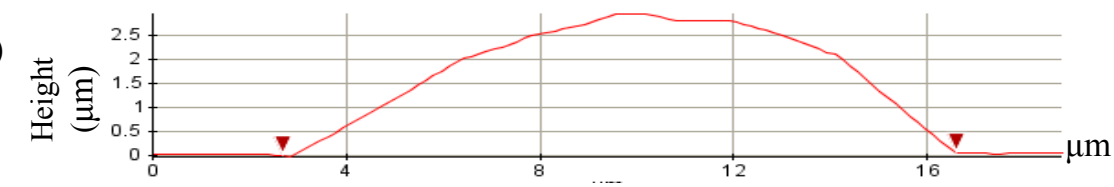

Figure 7: a) In-flight droplet before impact the substrate. b) Initial impact of the droplet on substrate. c) Droplet spreading on substrate surface. d) Droplet settlement and solidification. e) Experimentally measured droplet cross section on substrate. (Unit of $\mathrm{x}$ and $\mathrm{y}$ coordinates is $\mu \mathrm{m}$, and measurement is shown as red line).

which is shown in Figure 7(a); and the final state in the model is that droplet have completely solidify, which is shown in figure 7(d). The critical parameter in this process is the solidification time. If simulation time is shorter than the solidification time, the FEA results cannot get correctly. It is very difficult to precise calculate droplet solidification after impact to the substrate, but we can use equation $\tau_{s} \approx \frac{2 a^{2} k}{3 \alpha k_{t}}\left(\operatorname{In}\left(\frac{T_{0}-T_{a}}{T_{f}-T_{a}}\right)+\left(1+\frac{k_{t}}{2 k}\right) \frac{L}{c\left(T_{f}-T_{a}\right)}\right)$ where $a$ is the droplet radius, $\alpha$ is the thermal diffusivity, $k$ is the thermal conductivity, TO is the melting droplet temperature, $T_{a}$ is substrate temperature, $T_{f}$ is fusion temperature, $c$ is the melts specific heat and $\mathrm{L}$ is the latent heat of fusion, to
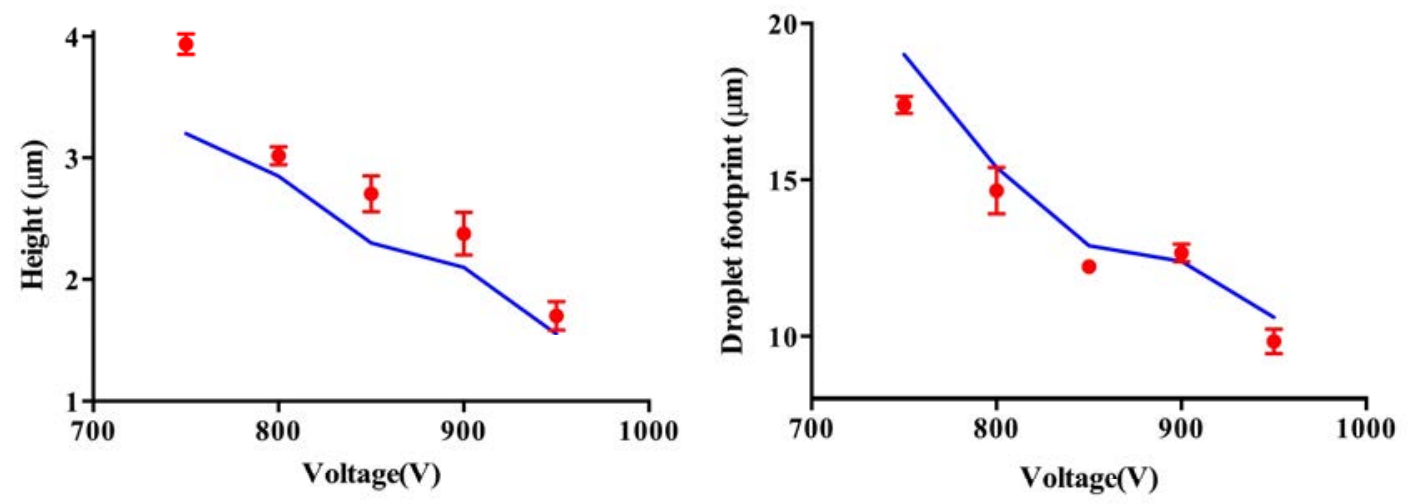

Figure 8: Comparison between droplets height (a) and diameter (b) trom FEA (lines) and experimentally measured results (data points) at different process conditions. 
roximately estimate it solidification time (Gao 1994), which is about $12 \mu \mathrm{s}$. In the FEA simulation, we have set the total simulation time at least two times larger than the estimate solidification time, to ensure that the droplets have completely solidified and stabilized. For each droplet ejected at different conditions, droplet impact velocity is obtained from the pervious model, as shown in the figure 6(e). Figure 8 shows the modeling result at the four stages of droplet settlement and an experimentally measured droplet cross-section using Atomic force microscopy (AFM). Figure 8 shows the droplet dimension on the substrate obtained from the model and experimental measurements. There is a good agreement bewteen the model and experiments, which means the model we have developed can decribe the solidfication and stabilizting process very well, and this model can be used to estimate droplet settlement under other different conditions.

\section{Conclusion}

In this work, we have study and model the EHD printing process using a phase-change material (i.e. wax) for micro-scale additive manufacturing, which can print sub-10 $\mu \mathrm{m} 3 \mathrm{D}$ microstructures. We built process models for modeling droplet formation, impact and settlement in printing process by FEA to predict droplet size in different process conditions. To model the droplet formation, we focused on the two competitive force, surface tension force and electrostatic force. By balancing those two forces at droplet pending from the Taylor cone, the droplet in-flight diameter can be derived. Droplet impact velocity can be calculated numerically by counting the effect from the electrostatic field when the droplet travels to the substrate. Then this velocity is used to model the droplet settlement on substrate. We have measured the volume and droplet footprint under different printing conditions, and compare them with the model prediction, which have a good match between them. We have also demonstrated the capability of using phase-change ink (wax) to print 3D structure by using EHD printing approach. This printing method gives high resolution and high aspect of ratio 3D structures with sub-10 $\mu \mathrm{m}$ resolution.

\section{Acknowledgement}

This work was supported in part by the National Science Foundation under Grant Award CMMI1333775 and CBET-1344618.

\section{References}

Beaman J, Marcus HL, Bourell DL, Barlow JW, Crawford RH, McAlea KP. Solid freeform fabrication: a new direction in manufacturing. Kluwer Academic Publishers, 1997.

Collins RT, Jones JJ, Harris MT, Basaran OA. Electrohydrodynamic tip streaming and emission of charged drops from liquid cones. Nat Phys 2008; 4(2):149-54.

Collins RT, Sambath K, Harris MT, Basaran OA. Universal scaling laws for the disintegration of electrified drops. Proc Natl Acad Sci 2013; 110(13):4905-10.

Forbes TP, Degertekin FL, Fedorov AG. Electrohydrodynamics of charge separation in droplet-based ion sources with time-varying electrical and mechanical actuation. Journal of the American Society for Mass Spectrometry 2010;21(4): 501-510.

Gao F, Sonin AA. Precise Deposition of Molten Microdrops: The Physics of Digital Microfabrication. Proceedings of the Royal Society of London. Series A: Mathematical and Physical Sciences 1994; 444(1922), 533-554. 
Gibson I, Rosen DW, Stucker B. Additive manufacturing technologies: rapid prototyping to direct digital manufacturing. Springer, 2009.

Han Y, Wei C, Dong J, Super-resolution electrohydrodynamic (EHD) 3D printing of micro-structures using phase-change inks, Manufacturing Letters 2014; 2(4): 96-99.

Hutmacher DW, Sittinger M, Risbud MV. Scaffold-based tissue engineering: rationale for computeraided design and solid free-form fabrication systems. TRENDS in Biotechnology 2004; 22(7):354362.

Kim H, Song J, Chung J, Hong D. Onset condition of pulsating cone-jet mode of electrohydrodynamic jetting for plane, hole, and pin type electrodes. Journal of Applied Physics 2010; 108(10): 102804.

Kruth JP, Leu MC, Nakagawa T. Progress in additive manufacturing and rapid prototyping. CIRP Annals-Manufacturing Technology 1998; 47(2): 525-540.

Lee MW, Kim NY, Yoon SS. On pinchoff behavior of electrified droplets. Journal of Aerosol Science 2013; 57: 114-124.

López-Herrera JM, Popinet S, Herrada MA. A charge-conservative approach for simulating electrohydrodynamic two-phase flows using volume-of-fluid. Journal of Computational Physics 2011; 230(5): 1939-1955.

Melchels F, Domingos M, Klein TJ, Malda J, Bartolo PJ, Hutmacher DW. Additive manufacturing of tissues and organs. Progress in Polymer Science 2011; 37(8):1079-1104.

Mishra S, Kira B, Andrew AG, Ferreira PM, Rogers JA. High-speed and drop-on-demand printing with a pulsed electrohydrodynamic jet. J Micromech Microeng 2010; 20(9): 095026.

Park JU, Matt H, Jun K, Kira B, Kurt A, Mukhopadhyay DK, et al. High-resolution electrohydrodynamic jet printing. Nat Mater 2007; 6(10):782-9.

Poellmann MJ, Barton K, Mishra S, Johnson AJW. Patterned hydrogel substrates for cell culture with electrohydrodynamic jet printing. Macromol Biosci 2011; 11(9): 1164-8.

Tan J, Saltzman WM. Topographical control of human neutrophil motility on micropatterned materials with various surface chemistry. Biomaterials 2002; 23(15): 3215-3225.

Wang K, Cai L, Zhang L, Dong J, Wang S. Biodegradable Photo-Crosslinked Polymer Substrates with Concentric Microgrooves for Regulating MC3T3-E1 Cell Behavior, Advanced Healthcare Materials 2012; 1(3):292-301.

Wei C, Dong J. Direct fabrication of high-resolution three-dimensional polymeric scaffolds using electrohydrodynamic hot jet plotting. J Micromech Microeng 2013; 23(2):025017.

Wei C, Dong J. Hybrid hierarchical fabrication of three- dimensional scaffolds. J Manuf Processes 2014; 16(2):257-63.

Wei C, Dong J. Development and Modeling of Melt Electrohydrodynamic-Jet Printing of PhaseChange Inks for High-Resolution Additive Manufacturing J. Manuf. Sci. Eng. 2014; 136(6): 061010 . 